\title{
Near-Field Optical Mapping of Quantum Hall Edge States
}

\author{
H. Ito, ${ }^{1}$ K. Furuya, ${ }^{1}$ Y. Shibata, ${ }^{1}$ S. Kashiwaya, ${ }^{2}$ M. Yamaguchi, ${ }^{3}$ T. Akazaki, ${ }^{3}$ H. Tamura, ${ }^{3}$ Y. Ootuka, ${ }^{1}$ and S. Nomura ${ }^{1, *}$ \\ ${ }^{1}$ Institute of Physics, University of Tsukuba, Tennodai, Tsukuba, 305-8571, Japan \\ ${ }^{2}$ National Institute of Advanced Industrial Science and Technology (AIST), Umezono, Tsukuba, 305-8568, Japan \\ ${ }^{3}$ NTT Basic Research Laboratories, NTT Corporation, Morinosato-Wakamiya, Atsugi, 243-0198, Japan
}

(Received 16 June 2011; published 16 December 2011)

\begin{abstract}
We report on the mapping of quantum-Hall edge states by quasiresonant photovoltage measurements using a near-field scanning optical microscope. We have observed fine structures near sample edges that shift inward with an increase in magnetic field in accordance with the shift of the positions of the quantum-Hall edge states. We have found a transition from the weak disorder regime where compressibleincompressble strips are visible to the strong disorder regime where fluctuations smear out incompressible strips.
\end{abstract}

DOI: 10.1103/PhysRevLett.107.256803

PACS numbers: 73.43.-f, 68.37.Uv, 78.56.-a

Quantum-Hall edge states [1,2] are formed by the intersect of electrostatic potential with the Fermi level near the edge of the two-dimensional electron system (2DES) in a magnetic field. Screening leads to the formation of the region of constant electrostatic potential and of smoothly varying electron density, known as compressible strips, and the region of constant electron density with a finite energy gap $(\Delta)$ at the Fermi level, known as incompressible strips. In the vicinity of the edge, the screening is reduced due to a stepwise decrease in local electron filling factor $\nu_{L}$. The screening properties are strongly modulated spatially by the formation of quantum-Hall edge states. Because of disorder the existence of alternating strips of compressible and incompressible liquids is not a prerequisite for the quantum-Hall effect [3]. Efros argued that the ratio of the energy gap $\Delta$ and the energy scale of a random potential $W$ measures a condition for the observation of alternating strips of compressible and incompressible liquids [3].

The spatial distribution of quantum-Hall edge states is thus an interesting problem to explore. While the existence of the edge states was confirmed indirectly by transport measurements $[4,5]$, the condition for observation of the edge states and the details of the potential and density profiles are still unclear experimentally. Continuous effort has been made, therefore, to directly probe alternating strips of the edge states, for example, by scanning single electron transistors [6], subsurface charge accumulation [7], scanning force microscope [8-10], and scanning tunneling spectroscopy $[11,12]$. Scanning gate microscopy $[13,14]$ has been used to image electron flow from a quantum point contact in magnetic fields. Those methods detect spatial profiles of charge densities or electrostatic potentials and were unable to discriminate energy levels. The ability to access states at a specific energy is one of the advantages of local probe methods based on optical excitations [15-18]. Previous investigations, however, do not utilize most of this feature since the photon energy was above the band gap of the AlGaAs barrier layer. Moreover, the spatial resolution of the order of microns [15-18] was not sufficiently high to resolve the edge states.

Here we report on the mapping of quantum-Hall edge states by quasiresonant photovoltage (PV) measurements using a near-field scanning optical microscope that permits energy selectivity to probe the edge states. By tuning the optical excitation energy, one can select to excite only the edge region or both the edge and the bulk regions of 2DES. Furthermore, by using a near-field optical probe, a spatial resolution of about $100 \mathrm{~nm}$ has been achieved. These enable us to resolve fine structures characteristic to the quantum-Hall edge states.

The sample was a standard Hall-bar structure of a GaAs/AlGaAs modulation-doped single heterojunction (SHJ) with a density of $4.6 \times 10^{15} \mathrm{~m}^{-2}$ after illumination and a mobility of $178 \mathrm{~m}^{2} / \mathrm{V} \mathrm{s}$. The 2DES was located $90 \mathrm{~nm}$ from the surface. The width and the length of the Hall bar were 25 and $300 \mu \mathrm{m}$, respectively. Our near-field scanning optical microscope is equipped with a tube piezoscanner and an $X Y Z$ piezoelectric inertial sliding drive stage. The sample on the scanning stage was illuminated with a laser light through a double tapered optical fiber probe [19] under a shear force feedback control in a dilution refrigerator with a base temperature of $70 \mathrm{mK}$, as schematically shown in Fig. 1(a). The scanning range of the tube piezoscanner was $2.1 \mu \mathrm{m}$ at $200 \mathrm{mK}$. The local optical excitation power under the probe aperture with a diameter of about $100 \mathrm{~nm}$ was kept to be less than $1 \mathrm{nW}$ in order to avoid the generation of hot carriers. There were a total of six contacts fabricated to the Hall bar. A PV between contacts 1 and 2 at each end as shown in Fig. 1(a) was amplified by a differential preamplifier, and was measured synchronously with a lock-in-amplifier at $159 \mathrm{~Hz}$ modulated by an optical chopper. Four other contacts on each side of the Hall bar were floating. Measurements were performed at the sample temperature of $200 \mathrm{mK}$ unless otherwise denoted. Details of experimental setup can be 

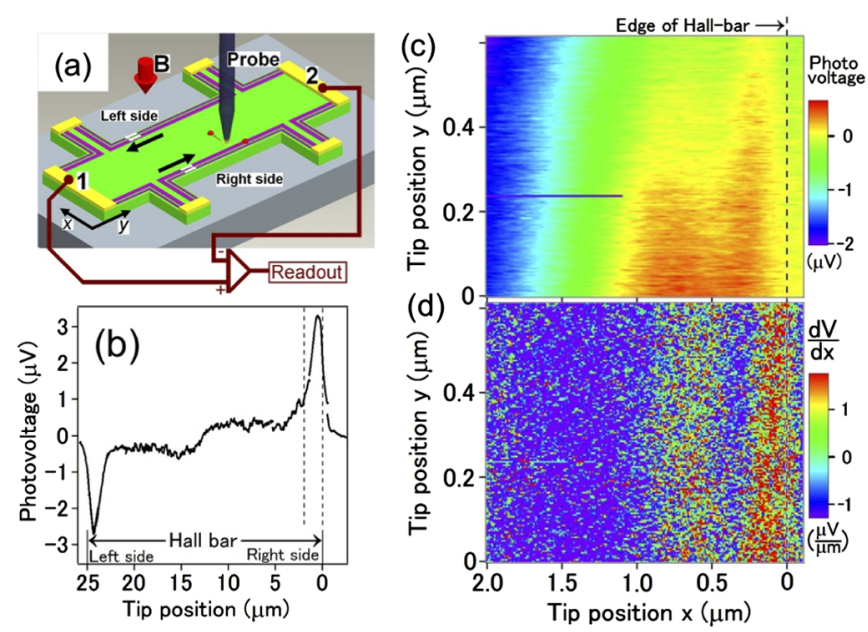

FIG. 1 (color online). (a) Schematics of the measurement setup and the sample structure. The arrows indicate the direction of electrons in the edge states. (b) A cross-sectional profile of photovoltage across a Hall-bar structure at $B=1.98 \mathrm{~T}(\nu=$ 9.55). (c) Mappings of PV near the edge of a Hall-bar structure and (d) their spatial derivative $d V / d x$ at $B=2.02 \mathrm{~T}(\nu=9.36)$, at the excitation energy of $1.5194 \mathrm{eV}$.

found elsewhere [20]. The change of the electron density due to local optical excitation was negligibly small because of the small excitation power and of the excitation energy lower than the band gap of the AlGaAs barrier layer. Hot carriers were also not generated electrically because the photocurrent was less than $1 \mathrm{pA}$.

A cross-sectional profile of PV across a Hall-bar structure is shown in Fig. 1(b) at magnetic field $B=1.98 \mathrm{~T}$ and electron filling factor $\nu=9.55$ at the excitation energy $\left(E_{p}\right)$ of $1.5194 \mathrm{eV}$ and the sample temperature $T=$ $200 \mathrm{mK}$. We take $B>0$ as magnetic field direction that penetrates from the front to the rear surface of the sample. The measurement was performed step-by-step by moving an inertial sliding drive stage to cover $25 \mu \mathrm{m}$ width of the Hall-bar structure. Large and sharp PV signals are observed near the edges with the polarity of PV signals different at opposite edges in agreement with the previous observations [16,18]. The position of the edge in Figs. 1(b)-1(d) was determined by a topographic image of the mesa structure. We checked that the PV profiles were not affected by reducing the excitation power by 2 orders of magnitude. The smaller and broader PV signals are observed in the bulk region of the Hall-bar structure. The sign of these PV signals changes in the middle of the Hallbar structure. Mappings of PV near the edge of the Hall-bar structure and their spatial derivative $d V / d x$ are shown in Figs. 1(c) and 1(d) at $B=2.02 \mathrm{~T}$. Fine structures in PV signals parallel to the edge are observed within $1 \mu \mathrm{m}$ from the edge. We show that these structures mark the formation of the alternating compressible and incompressible strips in what follows. We confirmed that the PV changes its sign in reversing $B$. Large $d V / d x$ is observed when the laser
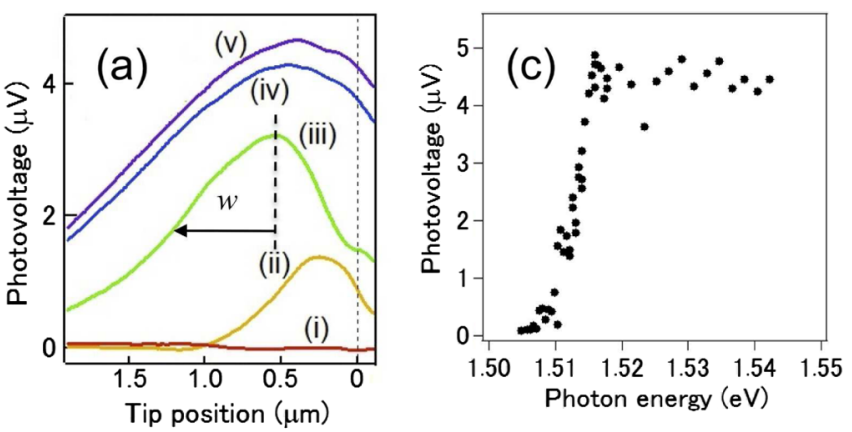

(b)
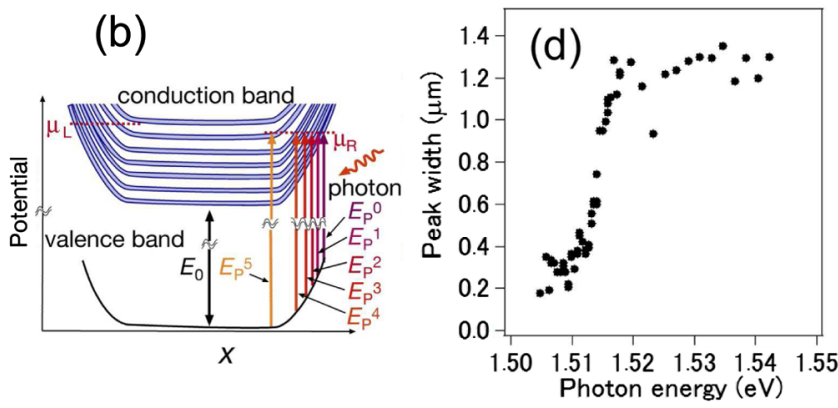

FIG. 2 (color online). (a) Optical excitation energy dependence of cross-sectional intensity profiles of PV at $B=1.64 \mathrm{~T}$ $(\nu=11.5)$ at the excitation energy of (i) 1.5083, (ii) 1.5120 , (iii) 1.5139, (iv) 1.5176, and (v) $1.5194 \mathrm{eV}$. (b) Schematic illustration of optical transitions between valence and conduction bands. The shaded area in the conduction band indicates broadened Landau levels. (c) Optical excitation energy dependence of peak PV at $B=1.64 \mathrm{~T}$. (d) Optical excitation energy dependence of half-peak width at $B=1.64 \mathrm{~T}$.

spot is located at the incompressible strip, where potential gradient in the $x$ direction is large, and positive and negative values of $d V / d x$ at $1 \mu \mathrm{m}<x<2 \mu \mathrm{m}$ are repeated at a period of $2 \nu$, as will be shown later. These observations agree with the Chklovskii, Shklovskii, and Glazman's (CSG) model [2]. Fluctuations of PV in the $y$ direction in Figs. 1(c) and 1(d) are due to disorder in the potential induced by the remote ionized donor distribution [21].

The optical excitation energy dependence of crosssectional intensity profiles of PV and that of peak PV are shown in Figs. 2(a) and 2(c), respectively. Measurement of electric conductance under monochromatic light irradiation is a well-established method to obtain the optical absorption spectrum [22]. PV signals are observed at the excitation energy above $1.5083 \mathrm{eV}$, which corresponds to the onset of the absorption of $\mathrm{GaAs} \mathrm{SHJ}\left(E_{0}\right)$ at the center of Hall bar. This indicates that the interband transition between the valence- and the conduction band is responsible for the PV signals. The PV increases, exhibits a peak at $1.5159 \mathrm{eV}$, and then saturates reflecting the twodimensional electron density of states with an increase in the excitation energy as shown in Fig. 2(c). When the tail of the absorption peak at $1.5120 \mathrm{eV}$ is optically excited, $\mathrm{PV}$ is observed only in the region within $0.5 \mu \mathrm{m}$ from the edge. As schematically illustrated in Fig. 2(b), there are six edge 
states at $B=1.64 \mathrm{~T}(\nu=11.5)$ where a bunch of five outer-edge states $\left(E_{p}^{0} \sim E_{p}^{4}\right)$ and the innermost-edge $\left(E_{p}^{5}\right)$ state can be probed separately by tuning the optical excitation energy [23] because the threshold energy for the optical transition is smaller at the outer-edge states $\left(E_{p}^{0} \sim E_{p}^{4}\right)$ than that at the innermost-edge state $\left(E_{p}^{5}\right)$. Thus the PV develops inwards with an increase in the excitation energy up to $E_{p}^{5}$ in the case of Fig. 2(b). The five outer-edge states are not resolved, partly due to the lifetime broadening of the energy of the optically created electron-hole pair that dissociates rapidly by the strong vertical electric field and partly due to the limitation of the spatial resolution. Half-width $w$ of PV is plotted as a function of photon energy in Fig. 2(d). It is seen that $w$ increases with an increase in the excitation energy and saturates at around $1.518 \mathrm{eV}$ as seen in Fig. 2(d). This behavior reflects the difference in the optical transition energies and the positions of the edge states. As the excitation energy increases, the number of the involved edge states increases from outer to inner edge states successively, and $w$ increases. When the excitation energy reaches up to $1.518 \mathrm{eV}$, the innermost-edge state with the highest optical transition energy finally contributes to the PV and then $w$ saturates. The optical excitation power dependence measurement shows that $w$ is nearly constant at 2 orders of magnitude of the excitation power. The observed change in the profiles of PV cannot be explained by the increase in carrier temperature. The profiles of $\mathrm{PV}$ in Fig. 2(a) thus probe the electrostatic potential near the edge, i.e., the softness of the confinement potential.

Temperature dependence of cross-sectional intensity profiles of PV is shown in Fig. 3 at the excitation energy

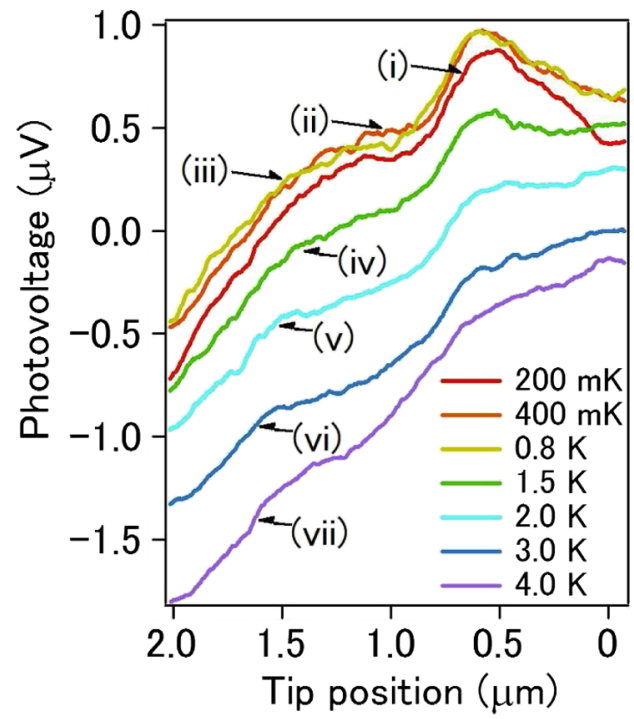

FIG. 3 (color online). Temperature dependence of crosssectional intensity profiles of $\mathrm{PV}$ at $B=2.02 \mathrm{~T}(\nu=9.36)$ at temperatures of (i) $200 \mathrm{mK}$, (ii) $400 \mathrm{mK}$, (iii) $0.8 \mathrm{~K}$, (iv) $1.5 \mathrm{~K}$, (v) $2.0 \mathrm{~K}$, (vi) $3.0 \mathrm{~K}$, and (vii) $4.0 \mathrm{~K}$, at the excitation energy of $1.5194 \mathrm{eV}$. of $1.5194 \mathrm{eV}$. Two features are seen: first, there are structures that can only be identified at low temperature near the edge, and second, the overall PV profile shifts to a negative direction with an increase in temperature. The structures in the PV profile which are only identified at a low temperature near the edge are most likely due to direct optical excitations to the quantum-Hall edge states $[1,2]$. These structures are smeared out with an increase in temperature. The overall shifts of the PV profile to a negative direction may be due to temperature dependence of $\sigma_{x x}$ and/or unknown temperature dependence of the voltage contacts.

In order to further confirm the above findings, we have investigated how mappings of PV develop with magnetic field. Figure 4(a) shows magnetic field dependence of mapping of the derivative of PV when the sample is excited at $1.5194 \mathrm{eV}$ above the first absorption peak in Fig. 2(c). It can be seen that two strips are observed at $B>1.94 \mathrm{~T}$, and they shift inward with an increase in $B$. We consider this region as a small fluctuation regime $(\Delta>W)$. The position of incompressible strips by the CSG model is given by $x_{k}=\frac{d_{0}}{1-\left(\nu_{L}\left(x_{k}\right) / \nu\right)^{2}}$, where $\nu_{L}\left(x_{k}\right)$ is a local filling factor, and $d_{0}=\frac{V_{0} a_{B}}{\pi E_{F}}$ is a depletion layer thickness. $d_{0}$ is estimated to be $134 \mathrm{~nm}$ by using $E_{F}=16.6 \mathrm{meV}, a_{B}=10 \mathrm{~nm}$, and $V_{0}=0.7 \mathrm{eV}$, where $V_{0}$ is taken to be the potential difference between 2DEG and an outside mesa. $x_{k}$ as plotted in Fig. 4(a) explains our observation well. Based on the Onsager-Casimir reciprocity theorem [24], the PV profile reflects the potential profile [18]. The derivative of the electrostatic potential shows a maxima at the incompressible strip. In the lower magnetic field of $B<1.58 \mathrm{~T}$, however, we cannot identify two strips. Moreover, an inward shift of PV profiles with an increase in magnetic field
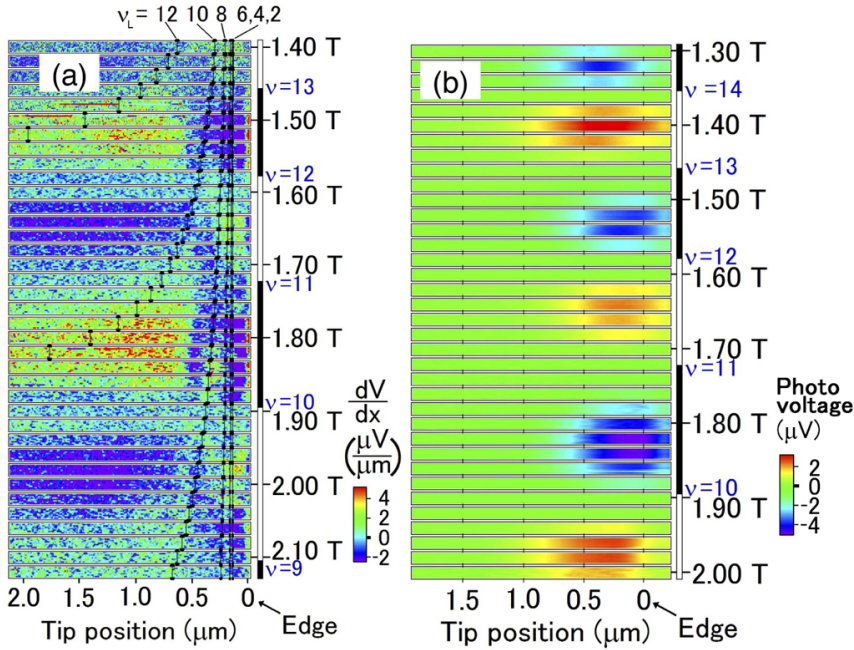

FIG. 4 (color online). (a) Magnetic field dependence of mapping of the derivative of PV near the edge of a Hall-bar structure at the excitation energy of $1.5194 \mathrm{eV}$. The position of incompressible strip $x_{k}$ is shown by vertical lines. (b) Magnetic field dependence of mapping of PV at the excitation energy of $1.5120 \mathrm{eV}$. The scanning range was $2.1 \mu \mathrm{m} \times 82 \mathrm{~nm}$. 
is unclear. We consider this region as a large fluctuation regime $(\Delta<W)$. The random potential due to fluctuations in donors is considered to smear out incompressible strips [3]. Our observations in Fig. 4(a) thus agree with the CSG model and the theory by Efros.

Figure 4(b) shows magnetic field dependence of mapping of PV when the sample is excited at $1.5120 \mathrm{eV}$ near the onset of the absorption. The PV signals appear only near the edge in all magnetic fields because only the outeredge states can be excited at $1.5120 \mathrm{eV}$. Surprisingly, Fig. 4(b) shows both positive and negative PV signals depending on $\nu$ and PV signals disappear at both even and odd $\nu$, unlike the results in the literature where the sign of the PV does not change when sweeping the magnetic field across the quantum-Hall minimum and vanishingly small PV was observed only at even $\nu$ [18].

In the case of $\nu$ slightly smaller than even numbers, positive $\mathrm{PV}$ is observed, the same sign of $\mathrm{PV}$ as in Figs. 1(b) and 2(a). The tunneling distance between the uppermost Landau level and the bulk states is small, and hence, the coupling between edge and bulk is large $[18,25,26]$. When the right side of the edge is illuminated, the optically created electrons is accelerated by the strong lateral electric field at the edge to the left. The electrons diffuse to the opposite left edge states by relaxing energy. The probability of the optically created electrons to reach the left edge states is larger than the probability of the electrons to be captured in the right edge states. Because the diagonal conductivity $\sigma_{x x}$ is small in the quantum-Hall effect, the Hall current balances the diffusion current. This results in a larger chemical potential at the left side than the right side [16]. The chemical potential at the right side is in equilibrium with the contact 2 as shown in Fig. 1(a) when the magnetic field penetrates from the front to the rear surface. The positive PV signals are thus explained.

In the case of $\nu$ slightly larger than even numbers, negative PV is observed as shown in Fig. 4(b). The tunneling distance between the uppermost Landau level and the bulk states is large, and hence, the coupling between edge and bulk is small $[18,25,26]$. Then the optically created electrons at the right side of the edge with small optical excess energy cannot diffuse across the bulk state. This induces a local increase of the electron density at the right side of the edge. This results in a larger chemical potential at the right side than the left side. The negative PV signals in Fig. 4(b) are explained by this model. These fine and alternating structures in PV have not been observed in the previous studies, since samples were illuminated by laser at the energy larger than the band gap of AlGaAs barrier layer [16-18]. In our measurement, very small excess energy of electrons, as well as high-resolution measurement, is an essential key to map the detailed structures of the edge states.

In summary, a high-resolution mapping of quantum-Hall edge states has been performed by locally creating electrons with small excess energies with a near-field scanning optical microscope. We have observed fine structures parallel to the edge in PV signals, which appear only at low temperature. We have shown that the optical excitation position can be selected by tuning the optical excitation energy. The $\nu$ dependent PV signals are explained by the oscillation of the tunneling distance between edge and bulk with $\nu$. Magnetic field dependence of mappings of PV shows both a large fluctuation regime where the inward shift of PV profiles is unclear and a small fluctuation regime where the fine structures shift inward with increase in $B$. Overall, we have demonstrated that it is possible to create electrons with controlled excess energy at an arbitrary position. We believe that direct mapping of the quantum-Hall edge states is a crucial step toward imaging of a fractional quantum-Hall phase [27] or a quantum anomalous Hall phase [28].

We appreciate fruitful discussions with Y. Hatsugai. This work was partly supported by Kakenhi DYCE No. 20104005, No. 20221007, and No. 21340076.

Note added in proof.-Recently, we became aware of related work by another group [29].

*snomura@sakura.cc.tsukuba.ac.jp.

[1] B. I. Halperin, Phys. Rev. B 25, 2185 (1982).

[2] D. B. Chklovskii, B. I. Shklovskii, and L. I. Glazman, Phys. Rev. B 46, 4026 (1992).

[3] A. L. Efros, Phys. Rev. B 60, 13343 (1999).

[4] B. J. van Wees, E. M.M. Willems, C. J.P. M. Harmans, C. W. J. Beenakker, H. van Houten, J. G. Williamson, C. T. Foxon, and J. J. Harris, Phys. Rev. Lett. 62, 1181 (1989).

[5] S. Komiyama, H. Hirai, M. Ohsawa, Y. Matsuda, S. Sasa, and T. Fujii, Phys. Rev. B 45, 11085 (1992).

[6] N. Zhitenev, T. Fulton, A. Yacoby, H. Hess, L. Pfeiffer, and K. West, Nature (London) 404, 473 (2000).

[7] S. Tessmer, P. Glicofridis, R. Ashoori, L. Levitov, and M. Melloch, Nature (London) 392, 51 (1998).

[8] K. L. McCormick, M. T. Woodside, M. Huang, M. Wu, P. L. McEuen, C. Duruoz, and J.S. Harris, Phys. Rev. B 59, 4654 (1999).

[9] P. Weitz, E. Ahlswede, J. Weis, K. von Klitzing, and K. Eberl, Physica (Amsterdam) 6E, 247 (2000).

[10] J. Hedberg, A. Lal, Y. Miyahara, P. Grtter, G. Gervais, M. Hilke, L. Pfeiffer, and K. West, Appl. Phys. Lett. 97, 143107 (2010).

[11] M. Morgenstern, J. Klijn, C. Meyer, M. Getzlaff, R. Adelung, R. A. Römer, K. Rossnagel, L. Kipp, M. Skibowski, and R. Wiesendanger, Phys. Rev. Lett. 89, 136806 (2002).

[12] S. Becker, C. Karrasch, T. Mashoff, M. Pratzer, M. Liebmann, V. Meden, and M. Morgenstern, Phys. Rev. Lett. 106, 156805 (2011).

[13] N. Aoki, C. R. da Cunha, R. Akis, D. K. Ferry, and Y. Ochiai, Phys. Rev. B 72, 155327 (2005).

[14] N. Paradiso, S. Heun, S. Roddaro, L. Pfeiffer, K. West, L. Sorba, G. Biasiol, and F. Beltram, Physica (Amsterdam) 42E, 1038 (2010). 
[15] R. J. F. van Haren, F. A. P. Blom, and J. H. Wolter, Phys. Rev. Lett. 74, 1198 (1995).

[16] A. A. Shashkin, A.J. Kent, J. R. Owers-Bradley, A. J. Cross, P. Hawker, and M. Henini, Phys. Rev. Lett. 79, 5114 (1997).

[17] H. van Zalinge, B. Özyilmaz, A. Böhm, R. W. van der Heijden, J.H. Wolter, and P. Wyder, Phys. Rev. B 64, 235303 (2001).

[18] H. van Zalinge, R. W. van der Heijden, J. H. Wolter, B. Özyilmaz, A. Böhm, and P. Wyder, Semicond. Sci. Technol. 19, 1153 (2004).

[19] T. Saiki, S. Mononobe, M. Ohtsu, N. Saito, and J. Kusano, Appl. Phys. Lett. 68, 2612 (1996).

[20] H. Ito, Y. Shibata, K. Furuya, S. Kashiwaya, Y. Ootuka, and S. Nomura, Phys. Procedia 3, 1171 (2010).

[21] M. Stopa, Phys. Rev. B 54, 13767 (1996).
[22] R. T. Collins, L. Vina, W. I. Wang, L. L. Chang, L. Esaki, K. v. Klitzing, and K. Ploog, Phys. Rev. B 36, 1531 (1987).

[23] S. Nomura and Y. Aoyagi, Phys. Rev. Lett. 93, 096803 (2004).

[24] H. Casimir, Rev. Mod. Phys. 17, 343 (1945).

[25] P. Svoboda, P. Středa, G. Nachtwei, A. Jaeger, M. Cukr, and M. Láznička, Phys. Rev. B 45, 8763 (1992).

[26] See Supplemental Material at http://link.aps.org/ supplemental/10.1103/PhysRevLett.107.256803 for details.

[27] C. W. J. Beenakker, Phys. Rev. Lett. 64, 216 (1990).

[28] Y. Zhang, Y.-W. Tan, H. L. Stormer, and P. Kim, Nature (London) 438, 201 (2005).

[29] Keji Lai, Worasom Kundhikanjana, Michael A. Kelly, Zhi-Xun Shen, Javad Shabani, and Mansour Shayegan, Phys. Rev. Lett. 107, 176809 (2011). 